\title{
Reduced-Complexity Radio Architectures for Enhanced Receive Selection Combining in Multiuser Diversity Systems
}

\author{
Elpiniki P. Tsakalaki, ${ }^{1,2}$ Osama N. Alrabadi, ${ }^{2}$ Constantinos B. Papadias, ${ }^{1}$ and Ramjee Prasad ${ }^{2}$ \\ ${ }^{1}$ Broadband Wireless and Sensor Networks Group, Athens Information Technology, 19002 Athens, Greece \\ ${ }^{2}$ Department of Electronic Systems, Aalborg University, 9220 Aalborg East, Denmark \\ Correspondence should be addressed to Elpiniki P. Tsakalaki, et@es.aau.dk
}

Received 1 August 2011; Revised 16 November 2011; Accepted 4 December 2011

Academic Editor: Li Yang

Copyright ( $) 2012$ Elpiniki P. Tsakalaki et al. This is an open access article distributed under the Creative Commons Attribution License, which permits unrestricted use, distribution, and reproduction in any medium, provided the original work is properly cited.

\begin{abstract}
Although antenna selection is a simple and efficient technique for enhancing the downlink performance of multiuser diversity systems, the large antenna interelement spacing required for achieving spatial diversity is prohibitive for user terminals due to size restrictions. In order to allay this problem, we propose miniaturized switched beam receiver designs assisted by low-cost passive reflectors. Unlike conventional spatial receive diversity systems, the proposed angular diversity architectures occupy a small volume whereas the antenna system properties are optimized by controlling the strong reactive fields present at small dimensions. The systems are designed for maximum antenna efficiency and low interbeam correlation, thus yielding $N$ practically uncorrelated receive diversity branches. The simulation results show that the proposed enhanced diversity combining systems improve the average throughput of a multiuser network outperforming classical antenna selection especially for small user populations and compact user terminal size.
\end{abstract}

\section{Introduction}

Recent studies have shown that the capacity-achieving strategy for the cellular downlink is dirty paper coding (DPC) at the multiple antenna base station (BS) [1]. A less computationally complex zero-forcing $(\mathrm{ZF})$ technique is proposed in [2] exhibiting an identical grow rate of the sum rate with respect to the number of user terminals (UTs). However, both DPC and ZF require perfect channel state information at the transmitter (CSIT). Since perfect CSIT is practically infeasible, the focus is on low-rate feedback techniques exploiting multiuser diversity by scheduling opportunistically transmissions to the users when their channel is good. These techniques, termed as opportunistic beamforming (OB), were introduced in [3]. Therein, taking into account fairness, the BS schedules in each time slot data transmission to the UT with the best normalized instantaneous channel condition relative to a random beamforming precoding. This approach achieves the asymptotic average throughput of coherent beamforming with modest signal-to-noise ratio (SNR) feedback. Several extensions to this technique are mentioned in the literature. Indicatively, [4] proposes an OB and scheduling algorithm that enhances the throughput and delay characteristics of the system whereas, in [5], multiple orthonormal random beams at the BS serve multiple UTs simultaneously in each time slot.

$\mathrm{OB}$ techniques rely on the assumption that, with a large number of UTs, there is a high probability that an arbitrary choice of a BS beamforming vector will be aligned to a user's channel vector [6]. Thus, the benefits of OB are more evident when the number of users is large. To further improve the data rates of multiuser diversity systems, especially for reasonable user populations, the authors in [69] consider multiple antenna UTs with different combining techniques ranging from the simple antenna selection combining (SC) to the more sophisticated equal gain combining (EGC), maximum ratio combining (MRC), and optimum combining (OC). However, complex architectures including 
multiple receive antennas are often prohibitive in UTs due to implementation complexity and size restrictions. Note that $\mathrm{SC}$ is the simplest combining method in terms of hardware complexity keeping the same diversity gain as that for MRC or EGC [9]. Moreover, the small spacing of the antenna elements at the UTs often results in significant correlation and coupling degrading the efficiency of the receive antenna system. Therefore, it is desirable to investigate simple to implement solutions ensuring low-power consumption and efficient performance at a constrained size.

In this paper, we propose two compact UT antenna architectures accompanied with SC aiming at enhancing the performance of a multiuser system in the downlink. The first antenna architecture consists of a simple switched uniform circular array (S-UCA) of $N$ antenna elements and a central common passive reflector (PR) terminated with a passive load (reactance) (Figure 1(a)). The second antenna architecture comprises a simple switched parasitic array (SPA) $[10,11]$ of a single RF chain circularly surrounded by $N$ PRs terminated with passive loads (Figure 1(b)). The two proposed architectures (S-UCA and S-PA) satisfy the aforementioned cost, size, and power restrictions as they both require a single RF chain and low-cost passive antenna hardware [12]. Moreover, the antenna size is kept small by reducing significantly the radius of the S-UCA and of the S-PA. In this way, the antenna systems retain strong beamforming capabilities due to the intense reactive fields egressed at such small spacing [13]. By optimizing the S-UCA and the S-PA loading conditions a high antenna efficiency is maintained whereas the $N$ beampatterns obtained by the receive architectures remain uncorrelated despite the small interelement spacing. Consequently, besides multiuser diversity, angular diversity is also made available resulting in significant multiuser performance gains compared not only to conventional single antenna UTs, but also to classical antenna SC with realistic receive antenna systems.

The two proposed antenna architectures preserve the compactness and cost constraints of UT antennas, whereas one can be thought of as the "dual" architecture of the other: the central element of the S-UCA is a PR surrounded by potentially active antenna elements (Figure $1(\mathrm{a})$ ), whereas in the case of S-PA the central element is an active antenna surrounded by a ring of PRs (Figure 1(b)). It should be noted that the S-PA necessitates a more complicated control circuit as compared to the S-UCA which requires a simple conventional antenna switching mechanism, resembling a classical switched UCA topology with the mere modification of inserting a common central PR. On the other hand, the RF switch needed for changing the RF path among the S-UCA antenna elements constitutes a liability in terms of additional insertion losses.

Notice that [14] proposes a compact single RF antenna system assisted by passive elements extracting a maximum diversity order of 3 (equal to the number of the considered basis beampatterns) in multiuser environments. However, the optimal antenna loading conditions are found according to the instantaneous SNR, thus adding extra computational complexity and delay. On the contrary, in our work the optimal loads are found off-line within a single-step design and remain fixed during communication. More important, tuning the antenna loads at such small spacing degrades the antenna efficiency, unless a dynamic matching circuit is assumed, thus complicating the whole system design. The efficiency degradation is an important issue addressed in this paper.

Another relevant previous work on miniaturized antenna systems with desired antenna properties is [15] describing a method to design a decoupling and matching network (DMN) for a set of preselected desired beampatterns and for compact antenna arrays. The present work is different from [15] in the sense that

(i) a single active antenna and, thus, a single radio are used in the proposed architectures compared to the all-active antenna system of [15];

(ii) the DMN with shunt connections between the ports in [15] results in significant bandwidth reduction, enhanced Ohmic losses, and, thus, smaller power gain compared to the type of uncoupled port matching of the proposed antenna systems $[16,17]$;

(iii) in the proposed work, we are able to express analytically the desired antenna properties in terms of antenna efficiency and diversity as function of the antenna loading conditions. This permits the proposed reduced-complexity radio architectures to be optimized for both antenna efficiency and angular diversity using a single optimization procedure (as described in Section 3). However, [15] follows a different approach according to which two iterative processes are required. The first iterative process aims at designing a DMN fulfilling the power conservation under a hypothetical lossless network assumption and certain desired port pattern properties whereas the second iterative process describes the creation of a realizable lossy DMN.

The following notations are used throughout the paper. $\mathbf{X}$ refers to a matrix and $\mathbf{x}$ refers to a vector of the specified size. $\|\mathbf{x}\|$ denotes the Euclidean norm of the vector $\mathbf{x}$, and $|Z|$ gives the amplitude of the complex number $Z$. $\mathbb{E}$ is the expectation operator and $\mathbb{C}$ denotes the set of complex numbers of the specified dimensions. The superscripts $T$ and $H$ denote transpose and transpose conjugate, respectively.

The rest of the paper is organized as follows. In Section 2, the system model is presented whereas Section 3 describes the proposed UT antenna design approaches. Section 4 encompasses various practical enhanced receive SC antenna design examples and their performance evaluation. Finally, Section 5 concludes the paper.

\section{System Model}

The downlink scenario of a single-cell multiuser wireless communication system is considered with one BS serving $K$ UTs. We assume the BS equipped with $M$ uncorrelated antennas whereas each of the UTs has $N$ beampatterns serving as $N$ virtual antenna elements. A narrowband, quasistatic fading channel model is considered so that the 


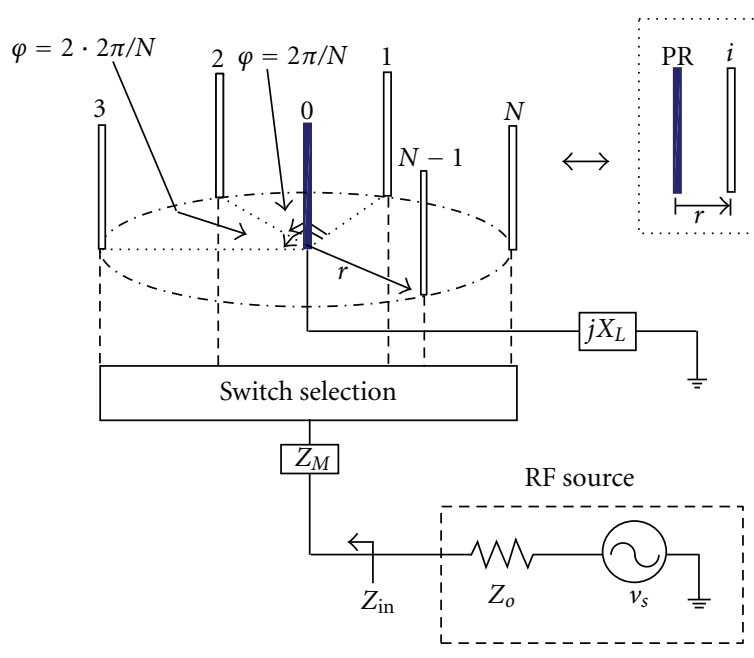

(a)

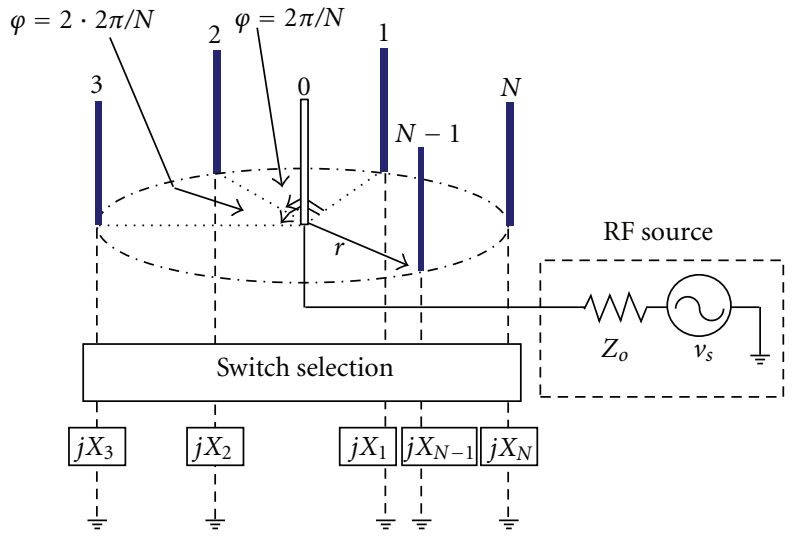

(b)

Figure 1: The proposed UT antenna configurations. (a) S-UCA of $N$ antenna elements surrounding a single passive reflector (element 0 ). (b) S-PA of $N$ passive reflectors surrounding a single active element (element 0 ).

channel response remains constant during one time slot, and then it abruptly changes to a new independent realization. At the beginning of each time slot, the base station is initialized subject to a random beamforming vector and retains its beamforming configuration constant over the slot. Collecting signals received from $N$ beams at the $k$ th UT into one vector $\mathbf{y}_{k} \in \mathbb{C}^{N \times 1}$, we get

$$
\mathbf{y}_{k}=\underbrace{(\eta \mathbf{R})^{1 / 2} \mathbf{H}_{w, k} \mathbf{w} s}_{\mathbf{H}_{k}}+\mathbf{n}_{k}=\left[\begin{array}{lll}
y_{k, 1} & y_{k, 2} \cdots y_{k, N}
\end{array}\right]^{T}
$$

where $s \in \mathbb{C}^{1 \times 1}$ is the transmitted symbol whereas the transmit power level is fixed at $P$ (i.e., $\mathbb{E}\left\{|s|^{2}\right\}=P$ ). $\mathbf{w} \in$ $\mathbb{C}^{M \times 1}$ is the transmit beamforming vector applied at the BS in a random fashion with unitary allocated power (i.e., $\left.\|\mathbf{w}\|^{2}=1\right) . \mathbf{n}_{k} \in \mathbb{C}^{N \times 1}$ is the noise vector at the $k$ th UT having independent and identically distributed (i.i.d.) circularly symmetric Gaussian entries with zero mean and variance $\sigma_{n}^{2}$, that is, $\mathbf{n}_{k} \sim \mathcal{C} \mathcal{N}\left(0, \sigma_{n}^{2} \mathbf{I}_{N}\right)$. Assuming closely spaced antennas on the receiver side and UTs equipped with identical antenna systems, the spatial correlation and the antenna efficiency are modeled by the receive correlation matrix $\mathbf{R} \in \mathbb{C}^{N \times N}$ and the efficiency of the UT antenna system $\eta \in[0,1]$, respectively. $\mathbf{H}_{k} \in \mathbb{C}^{N \times M}$ is the total channel matrix, $\left\{\mathbf{H}_{k}\right\}_{i, j}$ being the complex channel gain from the $j$ th antenna at the BS to the $i$ th antenna at the $k$ th UT, given by $\mathbf{H}_{k}=(\eta \mathbf{R})^{1 / 2} \mathbf{H}_{w, k}$. This equation results by applying the well-established Kronecker model [18] with single-sided (i.e., only receive) correlation, incorporating the receive antenna efficiency. $\mathbf{H}_{w, k} \in \mathbb{C}^{N \times M}$ has i.i.d. entries distributed as $\left\{\mathbf{H}_{w, k}\right\}_{i, j} \sim \mathcal{C} \mathcal{N}(0,1)$.

The equivalent single-input-multiple-output (SIMO) channel $\mathbf{h}_{k} \in \mathbb{C}^{N \times 1}$ at the $k$ th UT can be written as $\mathbf{h}_{k}=$ $\mathbf{H}_{k} \cdot \mathbf{w}=\left[h_{k, 1} h_{k, 2} \cdots h_{k, N}\right]^{T}$, where $h_{k, i}$ is the equivalent channel seen at the $i$ th antenna. Thus, the SNR perceived by the $i$ th antenna at the $k$ th UT is given by $\gamma_{k, i}=P\left|h_{k, i}\right|^{2} / \sigma_{n}^{2}$.

\section{Enhanced Selection Combining Antenna Architectures}

In this section we describe the two proposed antenna architectures for enhanced SC at the UT, that is, the SUCA (Figure 1(a)) and the S-PA (Figure 1(b)). The S-UCA and the S-PA are smart antenna systems that present a significant advantage over their classical all-active antenna array counterparts; they are able to control their beampatterns as any smart antenna system, while being implemented using a single RF chain. The suggested architectures are depicted with wire antenna elements in Figure 1. However, it should be noted that the following analysis is general regardless of the specific antenna element deployed and that the proposed designs accept any kind of antenna elements that can be practically integrated into compact UTs, such as printed monopoles, slot antennas, planar inverted-F antennas (PIFAs), and fractal antennas.

3.1. Switched Uniform Circular Array. The first proposed UT antenna architecture is a S-UCA comprising a ring of $N$ antenna elements (elements $1,2, \ldots, N$ in Figure $1(\mathrm{a})$ ) surrounding a central common PR (element 0 in Figure 1(a)) at relative local angles of $0,2 \pi / N, \ldots,(N-1) 2 \pi / N$, respectively. According to the antenna SC, only one antenna out of the $N$ available ones is active at each slot, that is, connected to the single RF chain via a switch and used for communication. The PR is short-circuited and loaded with a reactance $j X_{L}$ rather than being connected to the RF port. The PR is fed inductively by radiated energy coming from the active (driven) element. 
The $i$ th (active) antenna, $i \in\{1,2, \ldots, N\}$, has a corresponding beampattern $B_{i}(\varphi)$ given by

$$
B_{i}(\varphi)=\mathbf{i}^{T} \boldsymbol{\alpha}_{i}(\varphi),
$$

where $\varphi$ denotes the azimuthal angle representing the angle of arrival $(\mathrm{AoA}) . \boldsymbol{\alpha}_{i}(\varphi)$ is the $2 \times 1$ response vector given by

$$
\boldsymbol{\alpha}_{i}(\varphi)=\left[\begin{array}{ll}
1 & e^{-j \kappa r \cos [\varphi-(2 \pi / N)(i-1)]}
\end{array}\right]^{T},
$$

where $\lambda$ is the carrier wavelength and $\kappa=2 \pi / \lambda$ is the wavenumber. $r$ is the radius of the S-UCA corresponding to the spacing between each $i$ th antenna and the PR. Let $I_{A}$ and $I_{P}$ denote the current on each $i$ th antenna and the current induced on the PR, respectively. $\mathbf{i} \in \mathbb{C}^{2 \times 1}$ is the corresponding current vector, given by

$$
\mathbf{i}=v_{s}\left[\mathbf{Z}+\mathbf{Z}_{L}\right]^{-1} \mathbf{u}=\left[\begin{array}{ll}
I_{A} & I_{P}
\end{array}\right]^{T} .
$$

From (2)-(4) notice that the beampatterns $B_{i}(\varphi), i \in$ $\{1,2, \ldots, N\}$ are circularly symmetric to each other. In (4), $v_{s}$ represents the transmitted voltage signal source with the amplitude and the phase from the driven RF port whereas $\mathbf{u}=[10]^{T} \cdot \mathbf{Z} \in \mathbb{C}^{2 \times 2}$ is the mutual impedance matrix given by

$$
\mathbf{Z}=\left[\begin{array}{ll}
Z_{A A} & Z_{A P} \\
Z_{P A} & Z_{P P}
\end{array}\right]
$$

where $Z_{A A}$ and $Z_{P P}$ is the self-impedance of each $i$ th antenna and of the PR, respectively. $Z_{A P}=Z_{P A}$ is the mutual impedance between each $i$ th antenna and the PR. The loading matrix $\mathbf{Z}_{L} \in \mathbb{C}^{2 \times 2}$ can be defined as

$$
\mathbf{Z}_{L}:=\operatorname{diag}\left(\left[\begin{array}{ll}
Z_{o}+Z_{M} & j X_{L}
\end{array}\right]\right),
$$

where $Z_{o}$ denotes the terminal impedance at the active port (equal to the characteristic impedance of $50 \Omega$ ). $Z_{M}=R_{M}+$ $j X_{M}$ represents the matching impedance attached to the active element as an additional degree of freedom (besides $j X_{L}$ ) when optimizing the antenna system for efficiency and diversity.

Defining the row vector

$$
\mathbf{B}(\varphi):=\left[B_{1}(\varphi) B_{2}(\varphi) \cdots B_{N}(\varphi)\right]
$$

and assuming a uniform power angular spectrum (PAS), the correlation matrix $\mathbf{R}$, whose entry $\mathbf{R}_{i, j}$ denotes the correlation between the $i$ th and $j$ th beam, can be written as

$$
\mathbf{R}=\frac{1}{2 \pi} \int_{-\pi}^{+\pi} \mathbf{B}^{H}(\varphi) \mathbf{B}(\varphi) \cdot d \varphi,
$$

where $\mathbf{R}_{i, i}=1$ and $\mathbf{R}_{i, j}=\mathbf{R}_{j, i}, i, j \in\{1,2, \ldots, N\}$ by reciprocity. Notice that $\mathbf{R}$ is real valued for circularly symmetric beampatterns over a full angular spread [19]. Moreover, the structure of $\mathbf{R}$ is characterized by the expression

$$
\begin{aligned}
& \forall n \in\left\{1,2, \ldots,\left\lfloor\frac{N}{2}\right\rfloor\right\}, \quad i \in\{0,1, \ldots, N-2\}: \\
& \mathbf{R}_{1+(i \bmod N),[1+(i+n \bmod N)] \bmod N} \quad=\mathbf{R}_{2+(i \bmod N),[2+(i+n \bmod N)] \bmod N}
\end{aligned}
$$

which comes from the topology symmetry of any UCA and from the fact that pairs of equidistant antenna elements have the same correlation. The efficiency of the S-UCA is given by

$$
\eta=1-\left|\left(Z_{\text {in }}+Z_{o}\right)^{-1}\left(Z_{\text {in }}-Z_{o}\right)\right|^{2},
$$

where $Z_{\text {in }}$ is the driving point impedance seen by any active antenna such that

$$
Z_{\text {in }}=Z_{A A}+Z_{M}+\frac{I_{P}}{I_{A}} Z_{A P}=Z_{A A}+Z_{M}-\frac{Z_{A P}^{2}}{Z_{A P}+j X_{L}} .
$$

Notice that $Z_{\text {in }}$ remains constant for all $i \in\{1,2, \ldots, N\}$, and thus $\eta$ is maintained for any active antenna due to topology symmetry.

In order to find the optimal loading conditions that jointly maximize the S-UCA efficiency and minimize the maximum correlation among the available set of antennas, we solve the following optimization problem over the variable loads $Z_{M}$ and $X_{L}$ :

$$
\begin{aligned}
\underset{\left(\text { over } R_{M}, X_{M}, X_{L}\right)}{\operatorname{maximize}} & \eta \\
\text { subject to } & \max \left(\mathbf{R}_{i, j}\right) \leq 0.7, \quad i, j \in\{1,2, \ldots, N\}, \\
& 0 \leq R_{M} \leq R_{\mathrm{UB}}, \\
& X_{\mathrm{LB}} \leq X_{M}, X_{L} \leq X_{\mathrm{UB}} .
\end{aligned}
$$

We have constrained the magnitude of maximum correlation to be less than 0.7 , which is an empirical value at which diversity action takes place (see the detailed work in [19]). The constraints on $R_{M}, X_{M}$, and $X_{L}$ depend on the realizable range of the loads, where $R_{\mathrm{UB}}$ is the upper bound on $R_{M}$ whereas $X_{\mathrm{LB}}$ and $X_{\mathrm{UB}}$ are the lower and the upper bound on $X_{M}$ or $X_{L}$, respectively.

3.2. Switched Parasitic Array. The second proposed UT antenna architecture is a S-PA (see [10] and references therein). The single active element is surrounded by a ring of PRs where every PR is short-circuited and loaded with passive loads via on/off switches, rather than being connected to the RF port. A S-PA has as many possible main beampatterns as the number of PRs (given by $N$ ). The characteristics of the beampatterns (e.g., width, gain, and nulls) depend on the PRs loading and the array dimensions. The S-PA can switch among the $N$ beam positions by properly controlling the state of the switches. This operation can be performed very fast; for example, a PIN diode has a transient switching time in the order of nanoseconds [20].

The proposed S-PA comprises $N$ PRs (elements $1,2, \ldots, N$ in Figure 1(b)) surrounding the single active element (element 0 in the same figure) at relative local angles of $0,2 \pi / N, \ldots,(N-1) 2 \pi / N$. Similarly to the S-UCA, each of the $N$ beampatterns (in the far-field) is denoted by $B_{i}(\varphi), i \in\{1,2, \ldots, N\}$ and is given by

$$
B_{i}(\varphi)=\mathbf{i}_{i}^{T} \boldsymbol{\alpha}(\varphi) .
$$

The $n$th element of the S-PA $N \times 1$ response vector $\boldsymbol{\alpha}(\varphi)$ is given by $e^{-j \kappa r \cos [\varphi-(2 \pi / N)(n-1)]} . r$ is the spacing between the 
active element and the PRs and is equal to the radius of the S-PA. $\mathbf{i}_{i}$ is the vector of induced currents on the antenna elements, required for creating the $i$ th beampattern, given by

$$
\mathbf{i}_{i}=v_{s}\left[\mathbf{Z}+\mathbf{X}_{i}\right]^{-1} \mathbf{u}=\left[\begin{array}{llll}
I_{i, 0} & I_{i, 1} & \cdots & I_{i, N}
\end{array}\right]^{T},
$$

where $v_{s}$ represents the transmitted voltage signal source. $\mathbf{Z} \in$ $\mathbb{C}^{(N+1) \times(N+1)}$ is the mutual impedance matrix whose entry $Z_{m, m}, m \in\{0,1, \ldots, N\}$ is the self-impedance of the $m$ th antenna element, and $Z_{m, n}, m \neq n$ is the mutual impedance between the $m$ th and the $n$th antenna element (where $Z_{m, n}=$ $Z_{n, m}, m, n \in\{0,1, \ldots, N\}$ by reciprocity). The vector $\mathbf{u}$ is given by $\mathbf{u}=[1 \underbrace{1}_{N} \underbrace{0 \cdots 0}]^{T}$.

Let the loading vector $\mathbf{x}=\left[X_{1} X_{2} \cdots X_{N}\right] \Omega$ denote the set of $N$ loads to be mapped to the $N$ PRs, such that the circular rotation of the loading vector rotates the beampattern to one of the $N$ angular positions (based on the image theory). Then, the matrix $\mathbf{X}_{i} \in \mathbb{C}^{(N+1) \times(N+1)}$ can be defined as

$$
\mathbf{X}_{i}:=\operatorname{diag}\left(\left[Z_{o} j \hat{\mathbf{x}}_{i}\right]\right),
$$

where $\hat{\mathbf{x}}_{i}, i \in\{1,2, \ldots, N\}$, is a circular permutation of $\mathbf{x}$ at which the $i$ th beampattern is created and $\mathrm{Z}_{o}$ is the terminal impedance at the central active port. The $m$ th element of $\hat{\mathbf{x}}_{i}$

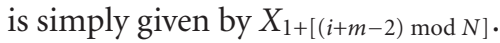

Similarly to the S-UCA, defining the row vector $\mathbf{B}(\varphi)$ as in (7) and assuming a uniform PAS, the $N \times N$ correlation matrix $\mathbf{R}$, whose entry $\mathbf{R}_{i, j}$ denotes the correlation between the $i$ th and $j$ th beampattern, is given by (8) whereas the SPA efficiency $\eta$ is given by (10). However, in the case of the $\mathrm{S}-\mathrm{PA}$, the driving point impedance seen by the central active element $Z_{\text {in }}$ is

$$
\begin{aligned}
Z_{\text {in }} & =Z_{0,0}+\sum_{m=1}^{N}\left(\frac{I_{i, m}}{I_{i, 0}}\right) Z_{0, i} \\
& =\frac{\mathbf{u}^{T} \mathbf{Z}\left(\mathbf{Z}+\mathbf{X}_{i}\right)^{-1} \mathbf{u}}{\mathbf{u}^{T}\left(\mathbf{Z}+\mathbf{X}_{i}\right)^{-1} \mathbf{u}} .
\end{aligned}
$$

$Z_{\text {in }}$ remains constant for all $i \in\{1,2, \ldots, N\}$ and thus $\eta$ is maintained for all $B_{i}(\varphi)$, due to topology symmetry.

In the S-PA case, the optimization problem yielding the set of the optimal reactive loads that jointly maximize the SPA efficiency and minimize the maximum correlation among the available set of beams over the reactance space $\mathbf{x}$ can be written as

$$
\begin{array}{ll}
\underset{\text { (over } \mathbf{x} \text { ) }}{\operatorname{maximize}} & \eta \\
\text { subject to } & \max \left(\mathbf{R}_{i, j}\right) \leq 0.7, i, \quad j \in\{1,2, \ldots, N\}, \\
& X_{\mathrm{LB}} \leq X_{m} \leq X_{\mathrm{UB}}, \quad m \in\{1,2, \ldots, N\},
\end{array}
$$

where $X_{\mathrm{LB}}$ is the lower bound and $X_{\mathrm{UB}}$ is the upper bound on the realizable range of the reactances $X_{m}$.

Comparing the optimization problems (12) and (17), we observe that the number of (real-valued) control variables is limited to 3 in the case of the S-UCA, whereas the S-PA has $2 \cdot N$ control variables. Thus, depending on the value of
$N$ (design parameter), the S-PA may have more degrees of freedom with respect to optimization flexibility compared to the S-UCA.

From hardware point of view, while the RF path is switched in case of the S-UCA, the RF path remains unchanged in the S-PA as the loads surrounding the central active element are rotated. Mathematically this has been represented by a constant current vector and a permuting steering vector in the S-UCA case, whereas a fixed steering vector and a permuting current vector express the S-PA switching mechanism.

3.3. Average Throughput. Since this work is focused on specific UT architectures and their impact on multiuser performance, a specific scheduling algorithm, such as proportional fair scheduling, is not considered. Within SC and assuming all UTs equipped with the described optimally loaded S-UCA or S-PA, the antenna element with the highest SNR $\gamma_{k, i}$ is selected to receive the signal for the $k$ th UT. Once the $k$ th UT selects the best beampattern and feeds back the corresponding SNR $\gamma_{k}^{*}=\max \left(\gamma_{k, 1}, \ldots, \gamma_{k, N}\right)$, the BS can schedule the user with the strongest $\gamma_{k}^{*}$. The resulting average throughput can be computed as [8]

$$
C=\mathbb{E}\left\{\log _{2}\left[1+\max _{k=1, \ldots, K}\left(\gamma_{k}^{*}\right)\right]\right\} \text {. }
$$

\section{Enhanced Selection Combining Antenna Design Examples and Performance Evaluation}

\subsection{Design Examples Using Thing Electrical Dipoles}

4.1.1. Throughput Performance. In this part we consider S-UCA set-ups of thin electrical dipoles vertical to the azimuth plane with $r=\lambda / 20$ and different number of antenna elements, that is, $N \in\{2,3,4,5,6\}$. The $\mathbf{Z}$ matrix is calculated using Gauss-Legendre numerical integration [21] obtaining $Z_{A A}=Z_{P P}=73.079+j 42.499$ and $Z_{A P}=71.607+j 24.251$. In order to solve the nonlinear optimization problem in (12), we utilize a constrained nonlinear optimization multivariate MATLAB routine [22]. We set $X_{\mathrm{LB}}=-100 \Omega$ and $X_{\mathrm{UB}}=100 \Omega$ as a feasible realizable range for $X_{M}$ or $X_{L}$ whereas $R_{M}$ is upper bounded by $R_{\mathrm{UB}}=100 \Omega$. The efficiency is found maximum $(\eta=1)$ for all S-UCA setups whereas the calculated optimal loads and correlation values are shown in Table 1 . The normalized magnitude of the obtained beampattern $B_{1}(\varphi)$ at the first antenna is illustrated in Figure 2 for the different S-UCA set-ups. The front-to-back ratio (FBR) of the beampatterns, defined as $20 \log _{10}\left|B_{1}(0) / B_{1}(\pi)\right|$, is also included in Table 1 which summarizes the favorable properties of the proposed S-UCA: maximum efficiency, low correlation values, and high FBR are available within a compact single radio UT antenna system.

In order to evaluate the SC via the proposed UT architecture, we consider the optimally loaded S-UCA with $N=6$ antennas. We compare to classical antenna SC 
TABLE 1: Optimized S-UCA characteristics $(\eta=1)$.

\begin{tabular}{|c|c|c|c|}
\hline$N$ & $\begin{array}{l}\text { Optimal loads } \\
(\Omega)\left\{R_{M}, X_{M}, X_{L}\right\}\end{array}$ & Correlation & $\mathrm{FBR}(\mathrm{dB})$ \\
\hline 2 & $\{45,-32,-3\}$ & $\mathbf{R}_{1,2}=0.0058$ & 9.2 \\
\hline 3 & $\{45,-8,-30\}$ & $\mathbf{R}_{1,2}=0.4752$ & 8.8 \\
\hline 4 & $\{45,-6,-32\}$ & $\begin{array}{l}\mathbf{R}_{1,2}=0.6000 \\
\mathbf{R}_{1,3}=0.2379 \\
\mathbf{R}_{1,2}=0.6000\end{array}$ & 10.6 \\
\hline 5 & $\{46,-27,-10\}$ & $\begin{array}{l}\mathbf{R}_{1,3}=0.0078 \\
\mathbf{R}_{1,2}=0.5942\end{array}$ & 5.3 \\
\hline 6 & $\{50,-8,-27\}$ & $\begin{array}{l}\mathbf{R}_{1,3}=0.2030 \\
\mathbf{R}_{1,4}=0.5935\end{array}$ & 6.3 \\
\hline
\end{tabular}

with the same number of diversity branches [23]. For this reason, we consider a conventional UCA of the same radius $r$ consisting of $N=6$ dipoles with the same self-impedance $Z_{A A}=79.33-j 19.65$. The UCA structure is similar to the proposed S-UCA structure of Figure 1(a), assuming the elements $1,2, \ldots, 6$ as the available set of antennas and omitting the central dipole (element 0 ). We assume each dipole matched for maximum efficiency $(\eta=1)$. The correlation is given by a real-valued $6 \times 6$ receive correlation matrix R. From [19] the correlation between any two antenna elements of the UCA assuming uniform PAS is given by $\mathbf{R}_{m, n}=(1 / 2 \pi) \int_{-\pi}^{+\pi} e^{j \kappa d^{(m, n)} \cos \varphi} \cdot d \varphi=J_{o}\left(\kappa d^{(m, n)}\right)$, where $d^{(m, n)}$ is the distance between the $m$ th and the $n$th antenna element, $m, n \in\{1,2, \ldots, 6\}$. In our case, $d^{(1,2)}=$ $2 r \sin ((2 \pi / 6) / 2), d^{(1,3)}=2 r \sin ((2 \pi / 3) / 2)$ and $d^{(1,4)}=$ $2 r \sin ((2 \pi / 2) / 2)$. Therefore, we get $\mathbf{R}_{1,2}=0.9755, \mathbf{R}_{1,3}=$ 0.9273 and $\mathbf{R}_{1,4}=0.9037$. As an upper bound on the achieved average throughput, we also consider the ideal case of SC with $\eta=1$ and $N=6$ fully uncorrelated diversity branches, that is, $\mathbf{R}_{i, j}=0, i \neq j, i, j \in\{1,2, \ldots, 6\}$.

From Figure 3 it is evident that SC with the proposed optimally loaded S-UCA provides significant increase to the average throughput as compared to the conventional singleantenna UTs as well as to classical antenna SC for $M=2$ and $P / \sigma_{n}^{2}=0 \mathrm{~dB}$. The observed gain for 3 users is $54 \%$ whereas classical antenna SC achieves only $17 \%$ with respect to single antenna UTs, revealing the significance of the proposed technique for small user populations. For 60 users, where the multiuser diversity gain dominates, the gain of SC with the proposed S-UCA decreases to $17 \%$, whereas the gain of antenna SC with conventional UCAs drops to 6.5\%. Notice that the performance of SC with the proposed practical antenna architecture almost achieves the throughput of the ideal case of 6 fully uncorrelated diversity branches.

Figure 4 shows the number of users required to achieve a target average throughput under different receive techniques $\left(M=2\right.$ and $\left.P / \sigma_{n}^{2}=0 \mathrm{~dB}\right)$. The figure shows that the SC with the proposed S-UCA requires almost one-sixth of the user population in order to obtain a certain performance

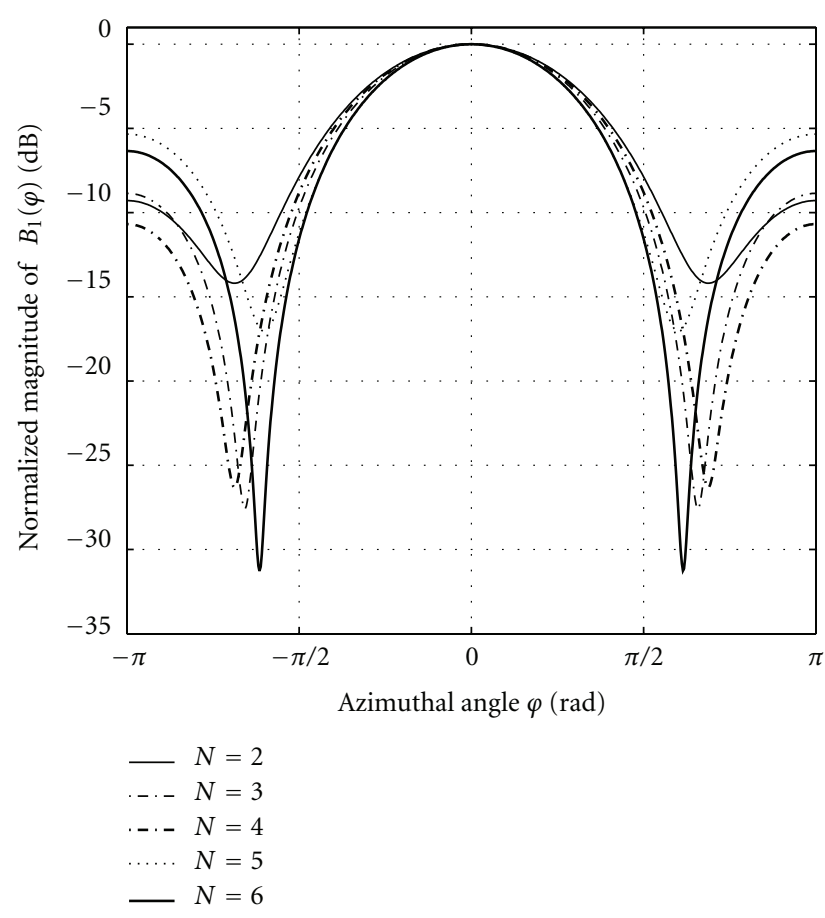

FIGURE 2: The normalized magnitude of the beampattern $B_{1}(\varphi)$ for the different $N$-element S-UCAs.

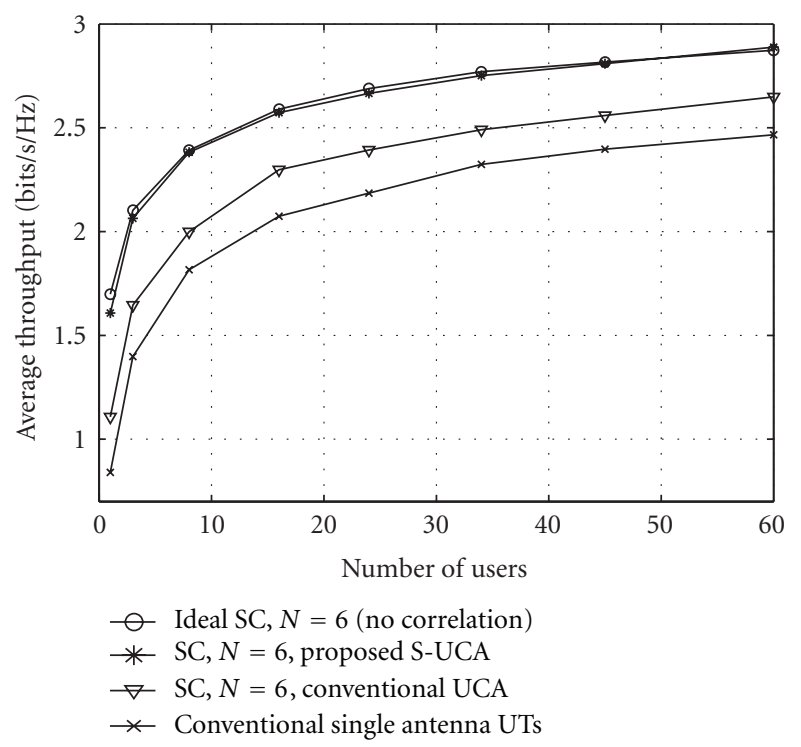

Figure 3: Average throughput as a function of the number of users.

compared to single antenna UTs. This confirms that SC represents an inherent form of multiuser diversity so that the degrees of freedom in the maximum selection increase from $K$ to $K \cdot N$ [7]. It should be noted that comparable performance gains of SC using the proposed S-PA compared to the classical antenna SC have been obtained as illustrated in the simulation results of [11]. 


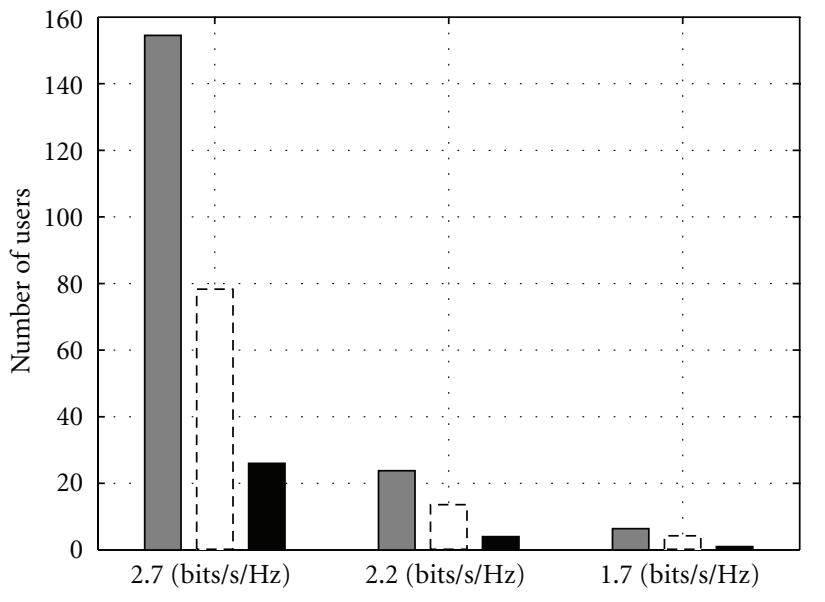

Conventional single antenna UTs

SC, $N=6$, conventional UCA

SC, $N=6$, proposed S-UCA

FIgURE 4: Number of users achieving a target average throughput.

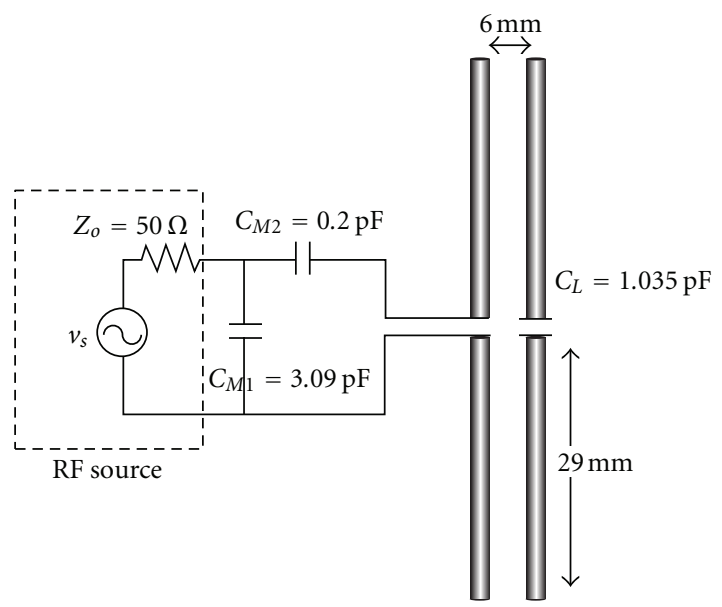

Figure 5: A four-element S-UCA design example comprising $60 \mathrm{~mm}$ long dipoles of $1 \mathrm{~mm}$ radius. At each time instant one dipole (out of the four) is active and closely coupled to the central passive one that is closed on a loading capacitor $C_{L}$.

4.1.2. Bandwidth Performance. The previous calculations are solely valid at the resonant frequency. In this part we aim at investigating the frequency response of the proposed antenna system. In general, the array bandwidth decreases as the mutual coupling level increases which is a natural phenomenon when considering closely spaced antenna elements where the strength of the reactive fields increases the quality factor of the antenna system. The problem worsens when decoupling the antennas using a DMN $[16,17]$. In this work, however, we perform a type of uncoupled matching (i.e., without any shunt connections between the ports) for maximum efficiency and low correlation. In order to examine the frequency response of the proposed enhanced receive diversity system we establish a well-defined

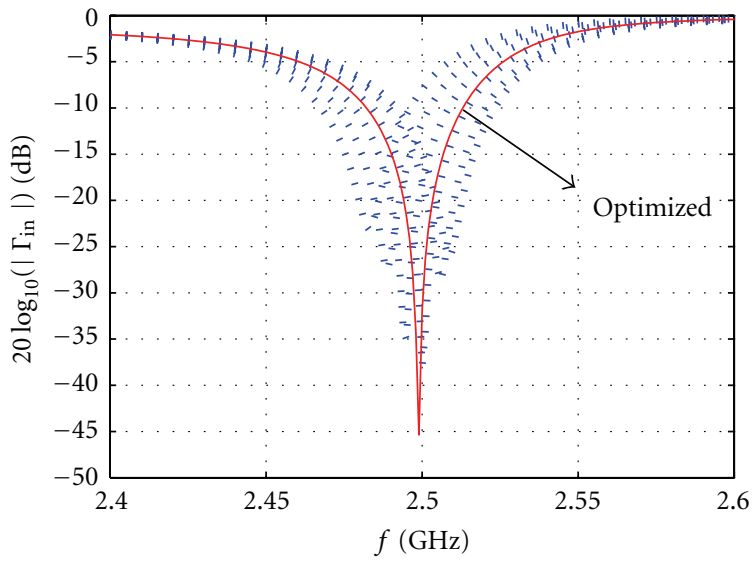

FIGURE 6: Frequency response of the four-dipole S-UCA design of Figure 5 .

design of a four-element S-UCA comprising $60 \mathrm{~mm}$ long dipoles, each having a radius of $1 \mathrm{~mm}$. A stand-alone dipole with these characteristics resonates at $f=2.5 \mathrm{GHz}$. A parasitic dipole (PR) with the same dimensions closed on a capacitor $C_{L}$ is placed at the center of the S-UCA (i.e., $\left.X_{L}=\left(2 \pi f C_{L}\right)^{-1}\right)$. Figure 5 shows the proposed S-UCA structure depicting only one out of the four potentially active dipoles in close proximity to the central PR. The RF source has a characteristic impedance $Z_{o}=50 \Omega$ and is assumed to be frequency independent and attached to the S-UCA through a L-section matching network of two capacitors $C_{M 1}$ and $C_{M 2}$, after which a switch selects one of the four dipoles. An ideal RF switch modeled as short and open circuit at the on and off state, respectively, is assumed. The impedance parameters of the antenna system were obtained by full-wave electromagnetic (EM) modeling, using the commercially available EM simulator IE3D. The $2 \times 2$ impedance matrix at the operating frequency of $2.5 \mathrm{GHz}$ is $\mathbf{Z}=\left[\begin{array}{cc}113.84+j 36.01 & 112.27-j 29.30 \\ 112.27-j 29.30 & 113.84+j 36.01\end{array}\right]$. The values of the matching capacitors and the loading capacitor $C_{M 1}, C_{M 2}$, and $C_{L}$, respectively, are obtained by solving the optimization formulation in (12) (the optimal values are shown in Figure 5) leading to a maximum correlation of 0.5983 and efficiency of $\eta=1$. The frequency response of this S-UCA at the optimal component values is shown in red color in Figure 6 which depicts the value of the input reflection coefficient $\Gamma_{\text {in }}$ in dB. The fractional bandwidth is $1.28 \%$ which maps to $32 \mathrm{MHz}$. It should be noted that an attempt to optimize the bandwidth has not been attained. Moreover, modern wideband systems requiring more bandwidth do not usually claim the whole band at one time; thus, the loading values can be tuned on demand in order to cover other bands as well.

Furthermore, in order to investigate the tolerance of the input return loss on the antenna loading, we varied $C_{L}$ over a $16 \Omega$ range in steps of $2 \Omega$ and obtained the frequency responses corresponding to the blue-dotted curves 


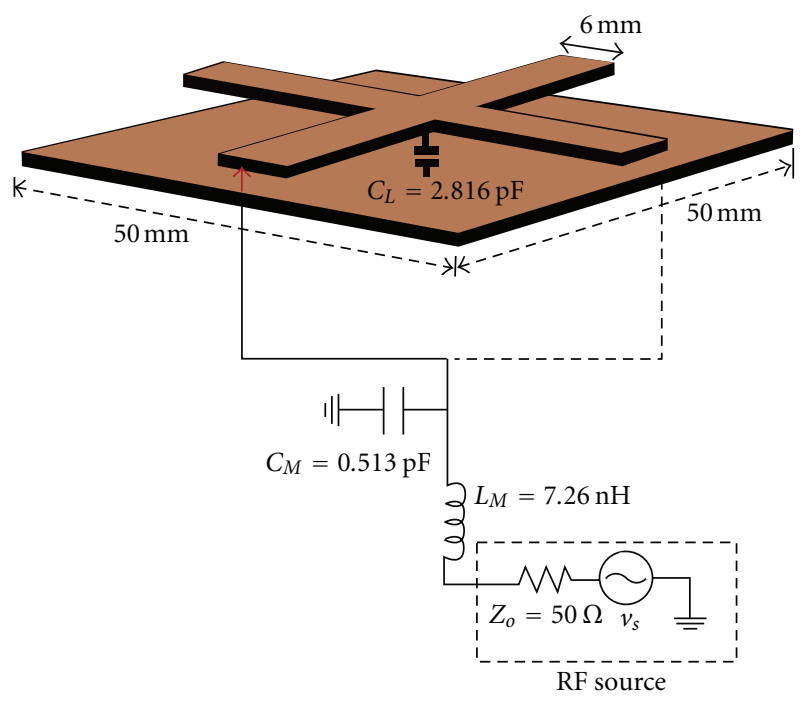

Figure 7: Practical S-UCA antenna design of four merged PIFA elements at $2.5 \mathrm{GHz}$.

in Figure 6. It is obvious from Figure 6 that the change in the load does not alter the operational bandwidth but it alters the central frequency. This, however, can be recovered by local handset calibration which is already required in practice to compensate for the user proximity effects [24].

\subsection{Design Examples Using Practically Integrable Antenna} Elements. A design example of a S-UCA with antenna elements that can be practically integrated into compact UTs is the four-modified PIFA antenna system shown in Figure 7, which has been designed on a $50 \mathrm{~mm} \times 50 \mathrm{~mm}$ printed circuit board (PCB) (almost half of the PCB area required for a bar-type phone chassis) operating at $f=2.5 \mathrm{GHz}$. The PIFA elements of this antenna system are modified in the sense that the excitation is done at the edge and the shorting is done in the middle of the element, whereas the inverse holds in a conventional PIFA element. The four antennas are merged over their common areas while a common shorting pin is terminated with a loading capacitor $C_{L}$ mounted on the antenna structure itself (i.e., $\left.X_{L}=\left(2 \pi f C_{L}\right)^{-1}\right)$. The dimensions of the antenna are given on the same figure.

Figure 8 shows how the proposed structure has evolved from two opposite modified PIFAs which are merged together. The operation is repeated on the second and fourth edges of the PCB. The RF source in Figure 7 has a characteristic impedance $Z_{o}=50 \Omega$ and is assumed to be frequency independent and attached to the S-UCA through a L-section matching network of a capacitor $C_{M}$ and an inductor $L_{M}$, after which a switch selects one of the four ports of the merged PIFA structure (an ideal RF switch model is also assumed here). The red arrow in Figure 7 indicates such a potentially active PIFA port. The correlation between the PIFA pairs was obtained by full-wave EM modeling, using the EM simulator IE3D. The reference port patterns (i.e., the radiation pattern of each port when excited by a unit voltage signal and the other ports are terminated with $50 \Omega$ ) at the

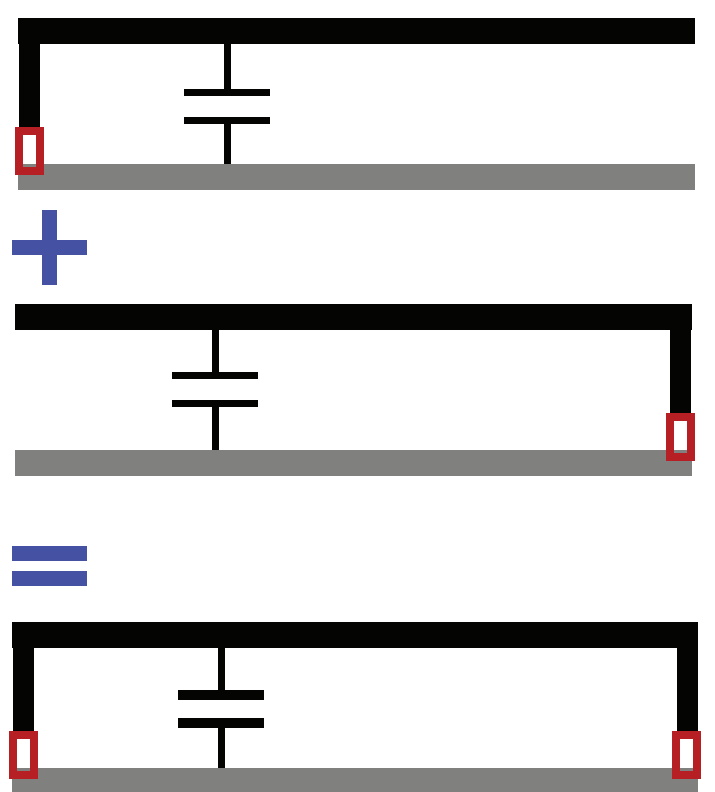

FIgure 8: Two opposite merged modified PIFA antennas comprising the building block of the proposed S-UCA structure of Figure 7.

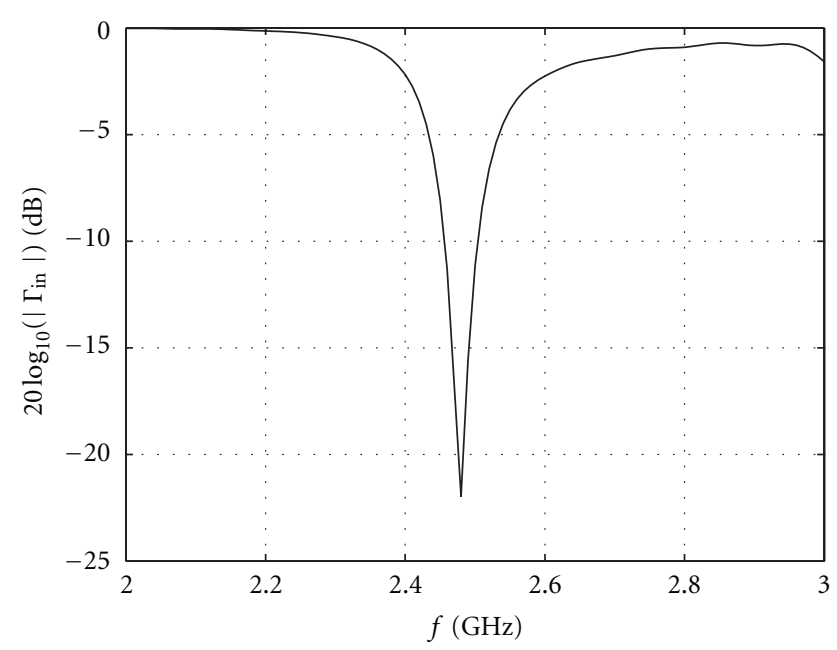

FIGURE 9: Frequency response of the practical receive diversity antenna design of Figure 7.

operating frequency of $2.5 \mathrm{GHz}$ were extracted and exported to a MATLAB routine out of which the correlation between the communication beams is found, in a procedure similar to the one in [25]. The $2 \times 2$ impedance matrix at $2.5 \mathrm{GHz}$ is obtained as $\mathbf{Z}=\left[\begin{array}{cc}17.88+j 382.60 & 1.06-j 29.71 \\ 1.06-j 29.71 & 1.13+j 25.12\end{array}\right]$. The L-section matching of $C_{M}$ and $L_{M}$ as well as the value of the loading capacitor $C_{L}$ is optimized for low correlation and maximum efficiency according to the optimization formulation in (12). The optimal values of the load $C_{L}$ and matching elements $C_{M}, L_{M}$ are given on Figure 7. The maximum correlation at the designated values in Figure 7 is found equal to 0.63 whereas the efficiency is $\eta=0.99$. The frequency response of the antenna is given in Figure 9. 
Another excellent example of extremely compact antennas for mobile terminals is element couplers [26] where the antenna is simply a matching component that excites the characteristic modes of the ground plane of the mobile device (i.e., the mobile device chassis modes) [27]. This type of antenna, namely, the switched-chassis mode system, is capable of exciting different weakly correlated modes acting as virtual uncorrelated antennas. It should be noted that an analysis similar to the one proposed for the S-UCA or the SPA system can be also applied to the switched-chassis mode system.

\section{Conclusion}

The paper proposes SC via optimized realistic receive antenna architectures for compact low-cost UTs in multiuser environments. It has been shown that the proposed compact S-UCA or S-PA antenna systems are able to achieve performance gains in terms of average throughput comparable to widely spaced antennas, by properly optimizing the antenna loading conditions for both antenna efficiency and diversity.

\section{Acknowledgments}

This research work has been performed in the context of collaboration between Aalborg University/Center for TeleInfraStruktur and Athens Information Technology for the joint offering of a Doctoral Program. The work was supported by the Future and Emerging Technologies (FET) Programme within the Seventh Framework Programme for Research of the European Commission, under FET-Open Grant CROWN-233843. This work is an extension to and generalization of the material presented in [11].

\section{References}

[1] G. Caire and S. Shamai, "On the achievable throughput of a multiantenna Gaussian broadcast channel," IEEE Transactions on Information Theory, vol. 49, no. 7, pp. 1691-1706, 2003.

[2] T. Yoo and A. Goldsmith, "On the optimality of multiantenna broadcast scheduling using zero-forcing beamforming," IEEE Journal on Selected Areas in Communications, vol. 24, no. 3, pp. 528-541, 2006.

[3] P. Viswanath, D. N. C. Tse, and R. Laroia, "Opportunistic beamforming using dumb antennas," IEEE Transactions on Information Theory, vol. 48, no. 6, pp. 1277-1294, 2002.

[4] D. Avidor, J. Ling, and C. Papadias, "Jointly opportunisitic beamforming and scheduling for downlink packet access," in Proceedings of the IEEE Int'l Conference on Communication (ICC '04), Paris, France, June 2004.

[5] M. Sharif and B. Hassibi, "On the capacity of MIMO broadcast channels with partial side information," IEEE Transactions on Information Theory, vol. 51, no. 2, pp. 506-522, 2005.

[6] L. Zan and S. A. Jafar, "Combined opportunistic beamforming and receive antenna selection [cellular downlink applications]," in Proceedings of the IEEE Wireless Communications and Networking Conference (WCNC '05), vol. 2, pp. 10071011, March 2005.

[7] R. Bosisio and U. Spagnolini, "On the sum-rate of opportunistic beamforming schemes with multiple antennas at the receiver," in Proceedings of the IEEE International Conference on Communications (ICC '07), pp. 1048-1053, Glasgow, UK, June 2007.

[8] M. O. Pun, V. Koivunen, and H. V. Poor, "Opportunistic scheduling and beamforming for MIMO-SDMA downlink systems with linear combining," in Proceedings of the 18th Annual IEEE International Symposium on Personal, Indoor and Mobile Radio Communications (PIMRC '07), pp. 1-6, Athens, Greece, September 2007.

[9] C. H. Chang and Y. Lee, "Opportunistic beamforming systems with diversity combining," in Proceedings of the 7th International Conference on Information, Communications and Signal Processing (ICICS '09), pp. 1-5, Macau, China, December 2009.

[10] D. V. Thiel, "Switched parasitic antennas and controlled reactance parasitic antennas: a systems comparison," in IEEE Antennas and Propagation Society Symposium, vol. 3, pp. 32113214, June 2004.

[11] E. P. Tsakalaki, O. N. Alrabadi, C. B. Papadias, and R. Prasad, "Enhanced selection combining for compact single RF user terminals in multiuser diversity systems," in Proceedings of the IEEE Int'l Symposium on Personal, Indoor, and Mobile Radio Communications (PIMRC '10), pp. 951-954, Istanbul, Turkey, September 2010.

[12] R. Vaughan, "Switched parasitic elements for antenna diversity," IEEE Transactions on Antennas and Propagation, vol. 47, no. 2, pp. 399-405, 1999.

[13] E. P. Tsakalaki, O. N. Alrabadi, C. B. Papadias, and R. Prasad, "Spatial spectrum sensing for wireless handheld terminals: design challenges and novel solutions based on tunable parasitic antennas [Dynamic Spectrum Management]," IEEE Wireless Communications Magazine, vol. 17, no. 4, pp. 33-40, 2010.

[14] V. Barousis, A. G. Kanatas, N. D. Skentos, and A. Kalis, "Pattern diversity for single RF user terminals in multiuser environments," IEEE Communications Letters, vol. 14, no. 2, Article ID 5403617, pp. 151-153, 2010.

[15] J. Weber, C. Volmer, K. Blau, R. Stephan, and M. A. Hein, "Miniaturized antenna arrays using decoupling networks with realistic elements," IEEE Transactions on Microwave Theory and Techniques, vol. 54, no. 6, pp. 2733-2740, 2006.

[16] B. K. Lau, J. B. Andersen, G. Kristensson, and A. F. Molisch, "Impact of matching network on bandwidth of compact antenna arrays," IEEE Transactions on Antennas and Propagation, vol. 54, no. 11, pp. 3225-3238, 2006.

[17] O. N. Alrabadi, MIMO communication using single feed antenna arrays, PhD Dissertation, Aalborg Universitet, 2011.

[18] D. S. Shiu, G. J. Foschini, M. J. Gans, and J. M. Kahn, "Fading correlation and its effect on the capacity of multielement antenna systems," IEEE Transactions on Communications, vol. 48, no. 3, pp. 502-513, 2000.

[19] R. G. Vaughan and J. B. Andersen, "Antenna diversity in mobile communications," IEEE Transactions on Vehicular Technology, vol. 36, no. 4, pp. 149-172, 1987.

[20] D. M. Pozar, Microwave Engineering, John Wiley \& Sons, Hoboken, NJ, USA, 2005.

[21] S. J. Orphanides, Electromagnetic Waves and Antennas, Rutgers University, 2008.

[22] The Mathworks, Natick, Mass, USA, http://www.mathworks. $\mathrm{com} /$.

[23] M. K. Simon and M.-S. Alouini, Digital Communication Over Fading Channels, John Wiley \& Sons, 2005.

[24] R. Mohammadkhani and J. S. Thompson, "MIMO capacity improvement in the presence of antenna mutual coupling," 
in Proceedings of the 18th Iranian Conference on Electrical Engineering (ICEE '10), pp. 167-171, Isfahan, Iran, May 2010.

[25] O. N. Alrabadi, J. Perruisseau-Carrier, and A. Kalis, "MIMO transmission using a single RF source: theory and antenna design," IEEE Transactions on Antennas and Propagation, vol. 60, no. 2, pp. 654-664, 2012.

[26] W. L. Schroeder, P. Schmitz, and C. Thome, "Miniaturization of mobile phone antennas by utilization of chassis mode resonances," in Proceedings of the German Microwave Conference (GeMiC '06), no. 7b-3, Karlsruhe, Germany, 2006.

[27] R. F. Harrington and J. R. Mautz, "Theory of characteristic modes for conducting bodies," IEEE Transactions on Antennas and Propagation, vol. 19, no. 5, pp. 622-628, 1971. 

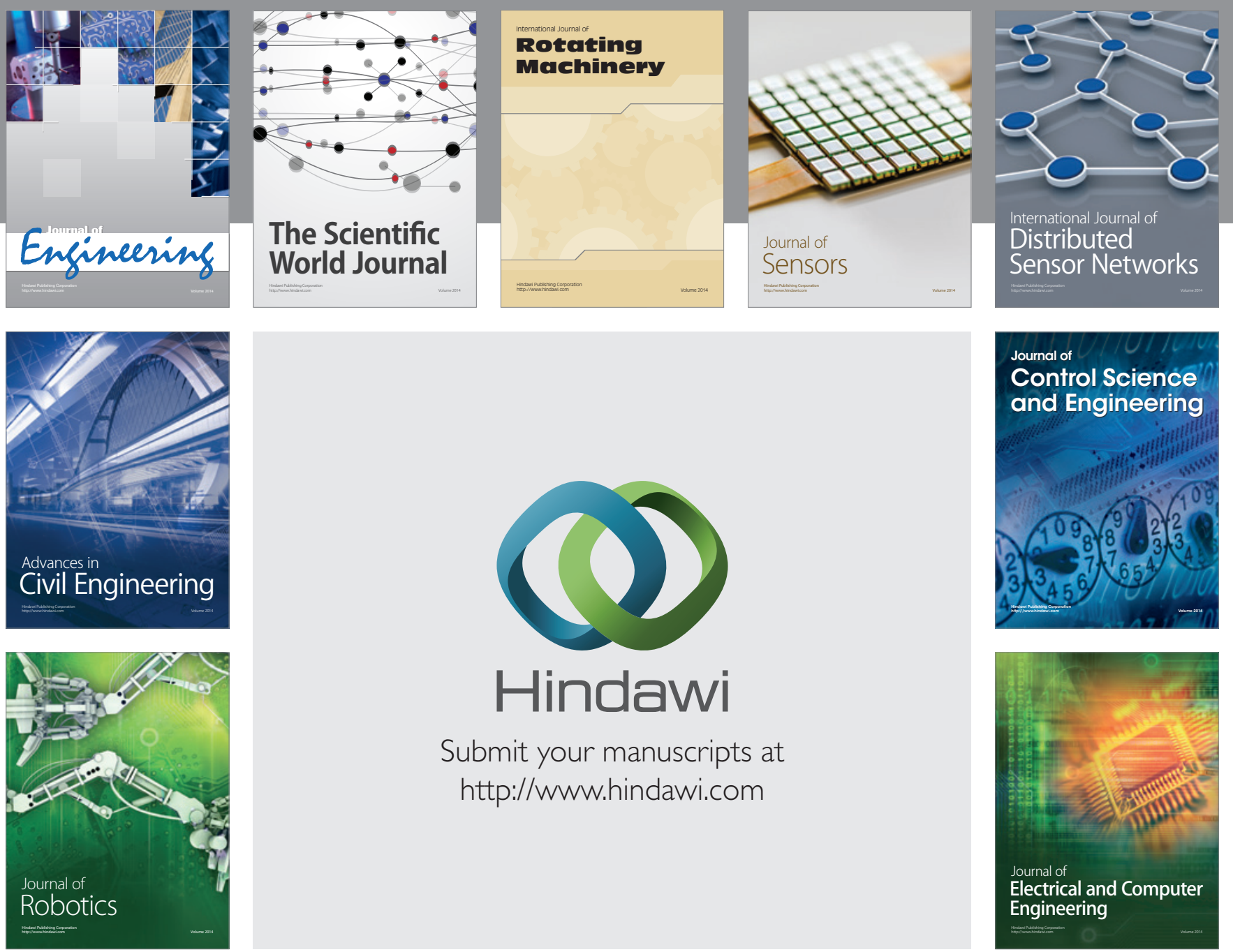

Submit your manuscripts at

http://www.hindawi.com
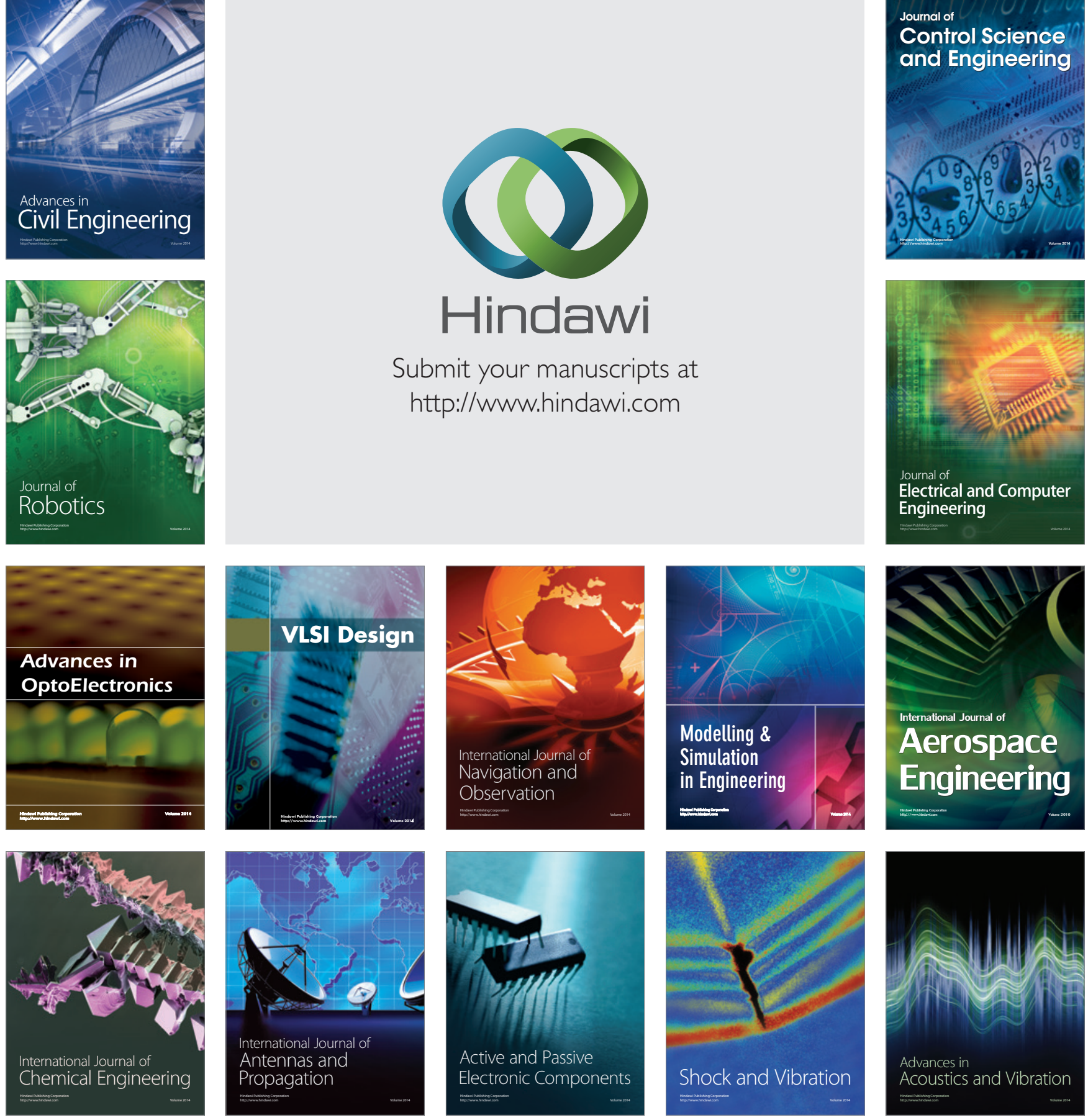